\title{
A NR2E1-Interacting Peptide of LSD1 Inhibits the Proliferation of Brain Tumor Initiating Cells
}

\section{Rong Hu}

The Sixth Affiliated Hospital of Sun Yat-sen University

\section{Umar Hameed}

National University of Singapore

\section{Xiang Sun}

The Chinese University of Hong Kong

\section{Balakrishnan Moorthy}

National University of Singapore

\section{Wen Zhang}

The Sixth Affiliated Hospital of Sun Yat-sen University

Philip Jeffrey

Princeton University

\section{Li Zhou}

The Chinese University of Hong Kong

\section{Xin Ma}

The Chinese University of Hong Kong

\section{Fangjin Chen}

Peking University

\section{Jianfeng Pei}

Peking University https://orcid.org/0000-0002-8482-1185

\section{Pankaj Giri}

Columbia University

\section{Yonggao Mou}

Sun Yat-sen University Cancer Center

\section{Kunchithapadam Swaminathan}

National University of Singapore

Ping Yuan ( $\nabla$ yuanp8@mail.sysu.edumcn )

The Sixth Affiliated Hospital of Sun Yat-sen University https://orcid.org/0000-0001-7243-3487

\section{Article}

Keywords: Brain tumor initiating cells, NR2E1, LSD1, Peptide, Brain cancer 
Posted Date: June 1st, 2021

DOl: https://doi.org/10.21203/rs.3.rs-492657/v1

License: (c) (i) This work is licensed under a Creative Commons Attribution 4.0 International License. Read Full License 


\section{Abstract}

The recurrence of malignant brain tumor, like glioblastoma, is often due to the existence of brain tumor initiating cells (BTICs) with stem cell properties. As the mortality ratio of brain tumor relapsed patients is very high and lack of efficient therapies, there is an urgent need to develop novel therapeutic methods targeting BTICs. NR2E1 (TLX), an orphan nuclear receptor, is critical for the self-renewal of BTICs. In this study, we found that NR2E1 recruits LSD1, a lysine demethylase, to demethylate mono- and di-methylated histone 3 Lys4 (H3K4me/me2) at the Pten promoters and repress its expression, thereby promoting BTIC proliferation. Using Amide Hydrogen/Deuterium Exchange and Mass Spectrometry (HDX-MS) method, we identified four LSD1 peptides that may interact with NR2E1. One of the peptides that locates at the LSD1 SWIRM domain strongly inhibited BTIC proliferation by promoting Pten expression through interfering NR2E1 and LSD1 function. Furthermore, overexpression of this peptide in human BTIC can inhibit its formation of brain tumor. Hence, this peptide exhibits an interesting potential for therapeutic intervention in malignant brain tumors in future.

\section{Introduction}

Malignant brain cancers, like glioblastoma (GBM), are highly heterogeneous and aggressive. They are resistant to chemotherapy and radiation therapy and show a high chance of relapse. The patient survival rate is only about 15 months after diagnosis. This situation has lasted over the past decades although multiple novel therapeutic means have been employed (Houshyari et al., 2015, Mrugala, 2013). It has been suggested that cancer initiating cells (CICs) with stem cell properties underlie the heterogeneity of malignant tumors (Zhu et al., 2014). They are less differentiated and resistant to chemotherapy and radiation treatment. They are thought to be the "root" of tumor occurrence and are responsible for the growth and relapse of tumors. Targeting $\mathrm{CICs}$ to treat cancer may help to improve the outcome of clinical therapies (Lin et al., 2013).

Researches have revealed that the recurrence of high-grade gliomas is due to the existence of brain tumor initiating cells (BTICs). BTICs were among the first CICs derived from a solid tumor (Singh et al., 2003, Singh et al., 2004). BTICs express the neural stem cell surface marker CD133. And as little as 100 BTICs could initiate phenocopies of the original tumors in a NOD.CB17-Prkdcscid/J (NOD SCID) mouse brain (Singh et al., 2004). BTICs and neural stem cells (NSCs) share several similarities, and it has been suggested that BTICs hijack the self-renewal mechanisms of NSCs to support their proliferation. Many studies have shown that the factors important for NSC maintenance also play important roles in brain tumorigenesis. For example, Nestin, which labels NSCs in adult mouse brain, also marks BTICs in glioblastoma and is required for the long-term sustenance of tumor growth (Chen et al., 2012).

NR2E1(TLX), an orphan nuclear receptor, is essential for the self-renewal of BTICs. NR2E1-positive glioma cells can initiate brain tumors and form spheres in suspension culture (Zhu et al., 2014). Depletion of 
NR2E1 in mouse primary tumors significantly extended animal survival time. Interestingly, GBM patients express a high level of NR2E1 which is correlated with poor survival time (Zhu et al., 2014). NR2E1 may therefore be a valuable target for brain tumor therapy. Just like Nestin, NR2E1 is highly expressed at the hippocampal dentate gyrus and the subventricular zone. It is required for the maintenance and selfrenewal of neural stem cells (Shi et al., 2004a). In NSCs, NR2E1 interacts with LSD1, a histone demethylase, and recruits it to the promoter of Pten. LSD1 then demethylates mono- and di-methylated histone 3 Lys 4 (H3K4) and removes these active epigenetic markers from the regions to silence the expression of Pten, a gene that induces apoptosis, regulates the cell cycle and functions as tumor repressors. Through coordinated repression of Pten, NR2E1 and LSD1 contribute to the proliferation of NSCs and retinoblastoma cells (Shi et al., 2004a, Yokoyama et al., 2008). Pten is an important misregulated tumor suppressor gene in almost all types of cancers. It is now an open and interesting question whether a similar mechanism is also employed in BTICs.

Lysine-specific histone demethylase LSD1 (also named AOF2 or KDM1A or BHC110) is a FAD dependent lysine demethylase. LSD1 can demethylate mono- and di-methylated H3K4 in a complex with CoREST, but shifts its targets to mono- and di-methylated H3K9 when it partners with the androgen receptor (AR) (Lee et al., 2006, Metzger et al., 2005, Shi et al., 2004b, Shi et al., 2005). Thus, by changing partners, LSD1 is involved in both gene activation and gene repression. The N-terminus of LSD1 is a non-structural element and contains a putative nuclear localization signal. Following this region is the Swip3p/Rsc8p/Moira (SWIRM) domain. After the SWIRM domain is an oxidase domain which is involved in demethylating proteins. LSD1 is also linked to the growth of glioblastoma and its inhibition increases the sensitivity of glioblastoma cells to histone deacetylase (HDAC) inhibitor treatment (Singh et al., 2011). Since NR2E1 and LSD1 both play important roles in glioblastoma, we set out to investigate whether BTICs employ the same NR2E1-LSD1 mechanism, as in NSCs, to regulate BTIC proliferation.

\section{Results}

\section{NR2E1 and LSD1 are essential for the proliferation of BTICs}

To study the role of NR2E1 and LSD1 in BTICs, two BTIC lines, BTIC-1 and BTIC-2 derived from Nestin-TVa mice were employed for the following experiments. These BTICs can efficiently grow mouse brain tumors after transplantation in recipient mice(Zhu et al., 2014). Like neural stem cells (NSCs), BTICs could be maintained in monolayer or non-adherent suspension culture (Figure 1a) (Zhu et al., 2014). The capacity of BTICs to form tumor spheres suggests their stem cell properties. NR2E1 and LSD1 are highly expressed in NSCs and promote the self-renewal of NSCs (Sun et al., 2010). Real-time PCR and western blot assays showed that both NR2E1 and LSD1 were more highly expressed in BTICs than NSCs (Figure $1 \mathrm{~b}$ and 1c). We therefore set to examine whether NR2E1 and LSD1 coordinate with each other to regulate the proliferation of BTICs. To knock down Nr2e 1 and $L s d 1$, we designed two different shRNAs for each gene and cloned them into pSuper-puro vector. We transfected these shRNAs in to BTICs respectively by electroporation and selected the transfected cells with puromycin(Sun et al., 2010). Three days after puromycin selection, Nr2e1 and Lsd1 shRNA knockdown led to a significantly lower amount of BTICs in 
culture (Figure 1d and Supplementary Figure 1a). Real-time PCR revealed that the expression of Nr2e1 and $L s d 1$ was downregulated to about $20 \%$ to $40 \%$ of their original levels by their respective shRNAs (Figure 1e and Supplementary Figure 1b). We then examined cell viability by the MTT cell proliferation assay, which revealed that the knockdown of $\mathrm{Nr} 2 e 1$ and $L s d 1$ resulted in reduced cell viability compared to the control knockdown BTICs (Figure $1 \mathrm{f}$ and Supplementary Figure $1 \mathrm{c}$ ). These results suggest that $\mathrm{Nr} 2 \mathrm{e} 1$ and Lsd1 are required for the proliferation of BTICs.

\section{NR2E1 and LSD1 synergistically repress PTEN to promote BTIC proliferation}

To examine whether NR2E1 and LSD1 play the same role in BTICs as in NSCs, we performed coimmunoprecipitation assay using whole cell lysate. An anti-NR2E1 antibody could pull down endogenous LSD1, but control IgG could not, suggesting that NR2E1 and LSD1 form a complex in BTICs (Figure 2a). To investigate whether NR2E1 and LSD1 also regulate Pten in BTICs, we knocked down the expression of Nr2e1 and Lsd1 in BTICs by shRNAs. The downregulation of NR2E1 and LSD1 led to the upregulation of PTEN at both the mRNA and protein levels (Figure $2 b$ and $2 c$, Supplementary Figure $2 a$ ). To examine whether PTEN is one of the major effectors of NR2E1 and LSD1, we generated shRNA that could efficiently downregulate Pten expression (Figure 2d). Downregulation of Pten expression in Nr2e1 knockdown BTICs or $L s d 1$ knockdown BTICs could rescue the cell death phenotype caused by only knockdown of $\mathrm{Nr} 2 \mathrm{e} 1$ or $\mathrm{Lsd} 1$ (Figure 2e-2f, Supplementary Figure 2b-2c). To further examine whether BTICs employed the same NR2E1-LSD1 regulatory mechanism as NSCs, we performed chromatin immunoprecipitation (ChIP) assay to investigate NR2E1 and LSD1 binding profile. The ChIP assay revealed that NR2E1 and LSD1 both bind to the promoter of Pten (Figure 2g). To examine whether NR2E1 and LSD1 are functional at the promoter of Pten, we performed ChIP assay with antibodies against H3K4me1 and H3K4me2 using chromatin extracted from Nr2e1 or Lsd 1 knockdown BTICs. It turned out that both downregulation of NR2E1 and LSD1 led to upregulated enrichment of H3K4me1 and H3K4me2 at the Pten promoter, suggesting that NR2E1 and LSD1 indeed directly repress Pten in BTICs by demethylating H3K4me and H3K4me2 at its promoter (Figure $2 \mathrm{~h}$ ).

\section{Prediction of Lsd1 peptides involved in the NR2E1-LSD1 interaction}

To understand how NR2E1 and LSD1 synergistically function, we employed Amide Hydrogen/Deuterium Exchange and Mass Spectrometry (HDX-MS) to investigate the interaction between NR2E1 and LSD1. A total of 56 pepsin digested fragments covering about $80 \%$ of the LSD 1 primary sequence were identified and analyzed. The difference in deuterium uptake for all the fragments between LSD1 alone and LSD1:NR2E1 complex was measured at 30 seconds, 1, 2, 5 and 10 minutes. As the difference in deuterium exchange was maximum at 1 minute, the deuterium uptake for each peptide in the 1 minute samples was used to monitor the effects of NR2E1 binding with LSD1. A number of regions in LSD1 showed decreased exchange upon interactions with NR2E1, but the maximum difference occurred at the regions within the AO domain. Peptides 333-350, 333-353 and 354-377 from the AO domain showed a significant decrease of 2.2, 2.5 and 4.0 deuterons, respectively (Figure 3a). Also, in the LSD1:NR2E1 complex, peptides 196-211, 197-211, 320-332, 333-344, 378-385, 419-441, 481-501, 498-511, 500-510, 
537-546, 601-614 and 623-650 from the SWIRM, Tower and AO domains showed a decrease of about 1.6 deuterons. Mass spectral isotope envelopes for four peptides, 197-211, 354-377, 481-501 and 537-546, from 1 minute HDX samples showed the most significant difference between LSD1 alone and NR2E1LSD1 complex after deuterium uptake, suggesting these LSD1 peptides may be involved in forming a complex with NR2E1 (Figure 3b). The difference in deuterium uptake for each peptide was calculated and the results from 1 minute samples were mapped onto the crystal structure of LSD1 (PDB ID: 2Z3Y) (Figure 3c).

The crystal structure of the NR2E1 ligand binding domain (LBD) has been solved (Zhi et al., 2015). NR2E1 LBD interacts with both the SWIRM and AO domains of LSD1 (Yokoyama et al., 2008). We have predicted the interaction between NR2E1 LBD (PDB code: 4XAI) and LSD1 (PDB code: 3ZMU) using the ZDOCK program (Chen et al., 2003). LSD1 residues 256-333, 410-435, 575-626, 712-744 and 792-819 that are not supposed to interact with NR2E1 were set as block residues. As a result, 3600 docking complexes were generated and clustered into 650 groups using the MMSTB clustering method (Feig et al., 2004) with a root-mean-square deviation (RMSD) cutoff at $8 \AA$. The buried solvent-accessible area, which is deemed as the possible binding surface of NR2E1 LBD by LSD1 peptides 197-211, 354-377, 481-501 and 537-546, was calculated by Naccess (Hubbard and Thornton, 1993) with a probe radius of $1.4 \AA$. LSD1 peptides 197-211, 354-377 and 481-501 individually showed varying predicted binding surface on NR2E1. However, LSD1-537-546 showed almost no predicted binding surface, suggesting that LSD1-537-546 is not likely involved in the interaction between LSD1 and NR2E1 LBD (Figure 3d).

\section{Role of LSD1 peptides in NR2E1-LSD1 interaction}

To further characterize the role of the LSD1 peptides 197-211, 354-377, 481-501 and 537-546 identified by HDX-MS, we generated Flag-tagged Lsd1 mutant clones by deleting the peptide encoding regions. These clone plasmids were then co-transfected with a plasmid expressing HA-NR2E1 into 293T cells for coimmunoprecipitation assay. Western blot revealed that while Flag-LSD1- $\Delta 197-211$, Flag-LSD1- $\Delta 354-377$ and Flag-LSD1- $\Delta$ 537-546 could be stably expressed, deletion of residues 481-501 of LSD1 however led to no detectable protein, suggesting this region is critical for the stable expression of LSD1 (Figure 4a). Then we performed immunoprecipitation with an anti-HA antibody using whole cell lysate and followed by immunoblotting with an anti-Flag antibody. It turned out that HA-NR2E1could pull down Flag-LSD1, FlagLSD1- $\Delta$ 354-377 and Flag-LSD1- $\Delta$ 537-546, but not Flag-LSD1- $\Delta 197-211$. This suggests that deletion of peptide LSD1-354-377 or LSD1 537-546 did not disturb the interaction between LSD1 and NR2E1, only deletion of LSD1-197-211 destroyed the NR2E-LSD1 complex (Figure 4a). Therefore, LSD1-197-211 is essential for NR2E1-LSD1 complex formation.

We next investigated the function of overexpression of these peptides in BTICs. We cloned the four peptides into the PCAG-puro plasmids and expressed them as Flag-tag peptides. Twelve hours after transfection, puromycin was added to select the transfected cells. At day 3 after puromycin selection, cells were harvested to check protein expression. Western blot with an anti-Flag antibody confirmed that all four Flag-tagged LSD1 peptides were expressed. Interestingly, the level of PTEN was increased in Flag- 
LSD1-197-211 overexpressed BTICs compared to GFP overexpressed BTICs (Figure 4b). Immunostaining BTICs at this stage with an anti-Ki67 antibody revealed that overexpression of LSD1-197-211 led to fewer Ki67 positive cells (Figure 4c). Further MTT assay confirmed that LSD1-197-211 transfected BTICs showed the most drastic reduction of viable cells, while LSD1-354-377 only slightly decreased BTICs and both LSD1-481-501 and LSD1-537-546 showed almost no effect (Figure 4d).

As transient express LSD1-197-211 could inhibit the self-renewal of BTICs, we next generated doxycycline inducible lentivirus to stably express LSD1-197-211 peptide-P2A-EGFP in BTICs. LSD1-197-211 peptide and EGFP are connected with P2A, a self-cleavage peptide囚Supplementary Figure 3a). After translation, LSD1-197-211 and EGFP are cut apart at P2A site. Hence区GFP protein level can reflect LSD1-197-211 level in transduced cells. We first infected 293T cells with these lentiviruse. After doxycycline induction for three days, it is clearly that both GFP lentivirus and LSD1-197-211-GFP lentivirus express similar level of GFP. Therefore LSD1-197-211 did not inhibit the growth of 293T cells (Supplementary Figure 3b). Next, we infected BTICs with the same batch of lentivirus and purified the transduced cells by puromycin selection. We then induced the peptide expression with doxycycline. 60 hours after induction, we performed immunostaining with antibody against cleaved Caspase-3, the marker of apoptosis. It turned out that BTICs that expressed LSD1-197-211 and GFP were often cleaved Caspase-3 positive, while it was not the case for the control GFP overexpressed BTICs (Supplementary Figure 4a). This result suggests that the expression of LSD1-197-211 in BTICs leads to apoptosis of the cells. Further extending the cell culture time, we observed that GFP positive LSD1-197-211 BTICs gradually lost their shape and detached from the plate. Eventually very rare GFP positive cells in LSD1-197-211 lentivirus transduced BTIC culture were observed (Supplementary Figure 4b). Analysis of GFP positive cell ratio with flow cytometry revealed that in contrast to the $78.6 \%$ GFP positive cells in the GFP lentivirus transduced BTICs, only $0.71 \%$ of the LSD1-197-211 lentivirus transduced BTICs showed weak GFP expression, suggesting that LSD1-197-211 peptide expressed BTICs were dead (Supplementary Figure. 4c). Soft agar colony formation assay also revealed that LSD1-197-211 overexpression drastically reduced the colony formation capacity of BTICs (Figure 4e). Furthermore, transwell assay revealed that overexpression of LSD1-197-211 drastically reduced the migration capacity of BTICs (Figure 4f). As expected, the size of tumor spheres formed by LSD1-197-211 overexpressed BTICs was smaller than GFP overexpressed BTICs (Figure 4g). Besides, the overall sphere number was also much less in LSD1-197-211 overexpressed BTICs than GFP overexpressed BTICs (Figure 4h), suggesting an inhibitory role of LSD1-197-211 on BTIC in vitro.

To examine whether the inhibitory effect of LSD1-197-211 on BTIC proliferation is due to the interference of NR2E1 and LSD1 synergistical function, we performed ChIP assay with antibodies against H3K4me1 and H3K4me2 with chromatin extracted from GFP, LSD1-197-211 and LSD1-354-377 overexpressed BTICs respectively. H3K4me1 and H3K4me2 modification at Pten promoter was significantly increased in LSD1-197-211 overexpressed BTICs, compared to the GFP overexpressed BTICs and LSD1-354-377 overexpressed BTICs (Figure 4i). These data suggest that LSD1-197-211 can inhibit BTIC proliferation by interfering the demethylation function of NR2E1-LSD1 complex on H3K4 methylation at Pten promoter.

\section{Specificity of peptide Lsd1-197-211}


Although LSD1-197-211 could interfere with the synergistic function of NR2E1 and LSD1 and inhibit the proliferation of BTICs, the specificity of this peptide is unclear. Both NR2E1 and LSD1 are highly expressed in 293T cells (Figure 5a). Knockdown of Nr2e1 by shRNA did not, however, lead to the upregulation of Pten at the mRNA level, suggesting that the NR2E1-LSD1 mechanism is not involved in the proliferation of 293T cells (Figure 5b). To test whether LSD1-197-211 had any effect on the cells that do not rely on the NR2E1-LSD1 based cell proliferation, we overexpressed GFP and LSD1 peptides in 293T cells separately with puromycin selection for three days. Western blot showed that the PTEN protein level was similar in the GFP and LSD1 peptide overexpressed BTICs (Figure 5c). MTT assay was further performed to check the cell viability. Apart from LSD1-354-377, which exhibited slight inhibition of 293T cell growth, other peptides showed no obvious inhibitory effect (Figure $5 \mathrm{~d}$ ). This result suggests that the LSD1-197-211 peptide shows relatively specific inhibition on BTICs.

To further characterize the specificity of LSD1-197-211, we determined the crystal structure of the human LSD1 SWIRM domain, residues 183-267 (Supp. Table 1). The SWIRM structure mainly contains a long central helix separating two smaller helix-loop-helix motifs at both sides (Figure 5e). The SWIRM domain highly resembles the SWIRM domain of previously determined human LSD1 crystal structure (PDB ID: 2Z3Y) (Supplementary Figure 5). Out of 687 aligned atoms, 528 atoms of the two structures can be well aligned with a root mean square of 0.418 . Unlike the SWIRM domain of SWI3 and ADA2, which binds to DNA, the SWIRM domain of LSD1 can neither bind to DNA nor does it contain any typical DNA-binding patch (Da et al., 2006). One major difference between LSD1 SWIRM domain and SWI3 and ADA2 SWIRM domains is mainly confined to the N-terminus. LSD1-197-211 is located at this region of the SWIRM domain, comprising a part of helix H2, a loop and a part of the long central helix H3 (Figure 5e). This region protrudes away from the hydrophobic core formed by $\mathrm{H} 6$ and the $\mathrm{AO}$ domain, which is involved in substrate demethylation (Chen et al., 2006). It forms a stable binding pocket with the $\mathrm{N}$ terminal loop and provides enough space to interact with other proteins (Figure 5e). Unlike LSD1 SWIRM domain, the corresponding region of ADA2 SWIRM is blocked by the N-terminal loop, making it impossible to interact with other proteins. For the SWI3 SWIRM domain, a big loop connects the $\mathrm{H} 2$ and $\mathrm{H} 3$ helices and no binding pocket is formed in this region (Figure 5e). The structural difference among LSD1, ADA2 and SWI3 SWIRM domains substantiates their different ligand binding properties.

\section{LSD1 197-211 inhibits the brain tumor formation of BTIC}

Human and mouse NR2E1 protein sequences share more than $97 \%$ similarity $\llbracket$ so does LSD1. In addition, human and mouse LSD1-197-211 peptides are exactly the same. Therefore, we deduced that LSD1-197211 peptide should be able to repress human BTICs (hBTICs) as well. To test this hypothesis, we derived two hBTIC lines from glioblastoma patients (Supplementary Figure 6a) and generated doxycycline inducible LSD1-197-211-GFP and control GFP transduced hBTICs by abovementioned lentivirus system. Next, we tested the effect of LSD1-197-211 on tumor sphere formation by adding doxcycycline to the culture medium. 3 days after doxycycline induction, LSD1-197-211-GFP transduced hBTICs formed slightly smaller tumor sphere than GFP transduced hBTICs. GFP signal in LSD1-197-211-GFP transduced hBTICs was also weaker than GFP transduced hBTICs. 6 days later, the difference was more drastic 
(Figure 6a and Supplementary Figure 6b). Not only the number of tumor spheres formed by LSD1-197211-GFP transduced hBTICs was less than GFP transduced hBTICs, but also the average tumor sphere size of LSD1-197-211-GFP transduced hBTICs was smaller than GFP transduced hBTICs (Figure 6b). These results confirmed that LSD1-197-211 inhibits hBTICs.

To further investigate the function of LSD1-197-211 in vivo, we transplanted the transduced hBTICs into the brains of nude mice. The next day after the transplantation, we started to feed the mice with water containing doxycycline to induce transduced gene expression. Mice transplanted with GFP transduced hBTICs showed obvious brain tumor growth as revealed by GFP positive signals, while almost no GFP positive signals were detected in mice transplanted with LSD1-197-211-GFP transduced hBTICs (Figure $6 \mathrm{c}-6 \mathrm{~d}$ ). Further Hematoxylin-eosin staining revealed obvious tumor infiltration in the brains of GFP transduced hBTIC transplanted mice, while most brains of mice transplanted with LSD1-197-211-GFP transduced hBTICs showed no or very small tumor infiltration (Figure 6e). By examining the genotype of the BTIC transplanted mouse brain via PCR, we confirmed that 11 mice were GFP hBTIC positive and 13 mice were LSD1-197-211-GFP hBTIC positive (Supplementary Figure 6c). The average brain tumor volume of LSD1-197-211-GFP hBTIC transplanted mice was obviously smaller than control BTIC transplanted mice (Supplementary Figure 6d). In addition, mice transplanted with GFP transduced hBTICs were much weaker and skinner than mice transplanted with LSD1-197-211-GFP transduced hBTICs (Supplementary Figure 6e-6f). Out of 11 GFP transduced hBTIC transplanted mice, 3 mice died before week seven after transplantation, while none of LSD1-197-211-GFP transduced hBTIC transplanted mouse died before week seven. Hence, LSD1-197-211 can suppress the tumor formed by hBTICs and improve the survival rate of hBITC transplanted mice.

\section{Discussion}

High-grade glioma, including glioblastoma, is the most common primary malignant brain tumor. The general treatment for high-grade glioma includes surgery, radiotherapy and chemotherapy. However, it is virtually impossible to completely resect these infiltrative tumors and concurrent radiotherapy and chemotherapy do not provide any significant survival benefit for patients. Five-year survival ratio of patient is still less than $5 \%$. Therefore, novel treatment strategies are desperately needed for this grim disease.

Past clinic research shows that drugs that target epigenetic modifiers yield promising survival benefits in multiple diseases. For example, the use of valproic acid, an HDAC inhibitor, together with radiotherapy, has shown a greater efficacy in GBM patients (Thotala et al., 2015, Weller et al., 2011). In recent years, LSD1 has been deemed as a very promising target, owing to its broad role in cancer, neurodegeneration and viral infection (Kashyap et al., 2013, Liang et al., 2013, Lynch et al., 2012, Schenk et al., 2012). However, no therapeutics targeting LSD1 have been developed at present. One of the reasons is that LSD1 is broadly expressed in mammalian tissues, in particular stem cells. Inhibition of LSD1 by AO inhibitors or depletion of LSD1 might therefore cause a significant disturbance of its normal physiological function, leading to unwanted side effects. All reported LSD1 inhibitors that bind to the FAD 
or AO domains are far from ideal, either because of poor selectivity or their polycationic nature (Lee et al., 2006, Schmidt and McCafferty, 2007, Willmann et al., 2012). Potential short peptides that could compete with natural histone $\mathrm{H} 3$ substrates or bind at sites beyond the active site of the AO domain by an allosteric mechanism to prevent LSD1 from forming complexes or binding to the nucleosomes are being actively explored (Baron and Vellore, 2012, Shi et al., 2005, Tortorici et al., 2013). Nevertheless, targeting the general LSD1 repressive complex, CoREST/LSD1 or LSD1 binding to the histone tail would still disturb the general function of LSD1 in cells. To achieve more effective and specific treatment of highgrade glioma, targeting the LSD1 involved specific cell proliferation mechanism is more appropriate, with minimum side effects.

Yokoyama et. al. have shown that in retinoblastoma cells the DNA binding domain (DBD) of NR2E1 interacts with the AO domain of LSD1, while the ligand-binding domain (LBD) of NR2E1 interacts with both the SWIRM and AO domains of LSD1 (Yokoyama et al., 2008). However, the details of these interactions are not reported. Our study has identified four possible NR2E1-interacting peptides of LSD1 using HDX-MS in vitro. Peptide deletion assay revealed that LSD1-197-211 peptide is critical for the interaction between NR2E1 and LSD1, since Flag-LSD1- 1 197-211 can no longer interact with NR2E1. To examine whether LSD1-197-211 and other identified peptides can synergistically interact with NR2E1, we investigated NR2E1 LBD/LSD1 docking complexes that showed more than $50 \AA^{2}$ solvent accessible area on NR2E1 by LSD1-197-211 and other identified peptides. It turned out that only LSD1-197-211 and LSD1354-377 may bind to NR2E1. However, in most docking complexes, the solvent accessible area of LSD1354-377 is smaller than that of LSD1-197-211, indicating that LSD1-354-377 may contribute to a weaker interaction between LSD1 and NR2E1 compared to LSD1-197-211 (Supp. Table 2). Indeed, compared to LSD1-354-377, which is located at the AO domain and shows only slight inhibition of the proliferation of both BTICs and 293T cells, LSD1-197-211 is located at the SWIRM domain and shows specific and potent inhibition of BTIC proliferation (Figure $4 \mathrm{~d}$ and Supplementary Figure 3b). Overexpression of LSD1197-211 efficiently blocks the function of NR2E1 and LSD1 complex and disrupts the LSD1 demethylation activity at the Pten promoter and leads to its upregulation, and therefore, inhibits the proliferation of BTICs (Figure 4).

However, since the NR2E1 and LSD1 mechanism is also employed by NSCs for self-renewal, we anticipate that LSD1-197-211 may also inhibit NSC proliferation. If this is the case, neurogenesis disturbance that might result from the use of this peptide for brain glioma treatment would be a concern. It is known that neurogenesis is most active in the fetus and reduces with aging. Elderly people, in whom neurogenesis is very low, have the highest probability of developing high-grade brain glioma. LSD1-197211 may therefore hold great therapeutic potential for patients of this age group. Besides, research has revealed that the regulatory factors of the self-renewal of NSCs in vitro do not always affect neurogenesis in vivo. For example, mice with mutated inhibitors of DNA binding 1 (Id1), a factor that is required for the self-renewal of NSCs in vitro, show normal neurogenesis and NSC population in vivo (Zhu et al., 2014).

Over all, our study revealed that Lsd1-197-211 may serve as a leading peptide for peptide drug development for glioblastoma. However, further investigation on the peptide delivery, safety and 
optimization will be needed to bring the discovery closer to application.

\section{Methods}

\section{Cell culture}

Brain tumor initiating cells (BTICs) derived from Nestin-TV-a mice were received as a gift from Dr. Haikun Liu's lab (Zhu et al., 2014). To culture BTICs in monolayer, the cell culture plate was coated with laminin and poly-L-lysine. Cells were grown in DMEM/F12 medium plus $20 \mathrm{ng} / \mathrm{ml}$ epidermal growth factor (EGF), $10 \mathrm{ng} / \mathrm{ml}$ fibroblast growth factor (FGF-2), B27 and insulin-transferrin-selenium supplements (ITSS). Cells were digested with accutase for passage. To culture brain tumor initiating cells in sphere, cells were seeded in a low attached cell culture plate (Corning) in the above medium without any coating.

\section{Cloning}

shRNA constructs were generated as previously described (Li et al., 2015). The sequences are as follows: Nr2e1 shRNA1 GGCTGTATCTGGCATGAAT囚shRNA2 CGTGGACACAAGGAAGACAAT囚Lsd1 shRNA1 CACAAGGAAAGCTAGAAGA, shRNA2 CCACAAGTCAAACCTTTATTT 『Pten shRNA CGACTTAGACTTGACCTATAT and control shRNA GATGAAATGGGTAAGTACA. The shRNAs were cloned into the pSuper-puro plasmid (Addgene) between the Bglll and Hindlll restriction sites. For transient gene overexpressing clones, NR2E1, LSD1 and LSD1 peptide cDNAs were cloned into the pCAG-puro plasmids. LSD1 peptide deletion clones were generated in the PCAG-puro vector by two-step PCR. For lentivirus vector construction, LD1-197-211 peptide cDNA was cloned into pLenti-TRE-EGFP-EF1-rtTA3-IRES-Puro plasmid at EcoRI restriction sites. The clone was sequenced and confirmed by forward primer ACGGTGGGAGGCCTATATAAGC and reverse primer CGTCGCCGTCCAGCTCGACCAG.

\section{Reverse transcription and real-time PCR}

Reverse transcription was performed with $2 \mu \mathrm{g}$ of total RNA using the PrimeScript RT reagent kit (Takara). Real-time PCR analysis was performed by using the ABI Prism 7900HT machine (Applied Biosystems) with the SYBR Green mixture (Takara). For each primer, only one correct size band was formed. All experiments were repeated at least three times independently. The final results were normalized against the expression of $\beta$-ACTIN or GAPDH.

\section{Cell growth assay}

$10^{6}$ BTICs were transfected with 2 ug plasmids of interests by electroporation with Amaxa cell line Nucleofector Kit V using nucleofector II. The transfected cells were seeded on a poly-L-lysine and laminin treated 6-well plate. After 24 hours, the transfected cells were selected with $1 \mathrm{ug} / \mathrm{ml}$ puromycin. After 3 days, the floating dead cells were washed away and the viability of cells was quantitated using MTT assay or CCK-8 assay kit by following manufacturer's protocol. 
ChIP assay was carried out as described previously with slight modification (Li et al., 2013b). Briefly, cells were fixed with $1 \%(\mathrm{w} / \mathrm{v})$ formaldehyde for 10 minutes at room temperature, and $125 \mathrm{mM}$ glycine was used to inactivate formaldehyde. Chromatins were sonicated to generate average fragment sizes from 200 to 600 bp and immunoprecipitated using the anti-NR2E1 (a gift from Liu's lab), anti-Lsd1 (ab17721), anti-H3K4me1 (ab8895), anti-H3K4me2 (ab7766) antibodies and control IgG or control GFP. The ChIP enriched DNA and input were then decrosslinked and proteins were digested by proteinase K. DNAs were purified by phenol:chloroform extractions and followed by ChIP-qPCR analysis using the ABI PRISM 7900 sequence detection system and Kappa SYBR green master mix (Takara). The values of each real-time PCR assay were normalized with its own input value and then compared with the IgG or GFP value to get the enrichment fold. PCR primers were designed to amplify the promoter regions of mouse Pten and control according to previous research(Sun et al., 2010). Each experiment was performed three times independently.

\section{Western blot}

Western blot was performed by following standard protocols (Li et al., 2013a). Total protein was collected by lysing cells with RIPA buffer containing $0.2 \mathrm{M} \mathrm{NaCl}, 1 \%$ SDS, $1 \mathrm{mM}$ PMSF and $0.1 \mathrm{M}$ DTT. Proteins were then separated by SDS-PAGE and transferred to PVDF membrane (Pall). The membrane was blocked with TBS $+0.1 \%$ Tween with $5 \%$ nonfat milk (BD) and then blotted with proper primary antibody in TBS $+0.1 \%$ Tween at cold room overnight. The primary antibodies and dilutions were: rabbit antiLSD1 (ab17721, Abcam) at 1/1000, rabbit anti-NR2E1 (a gift from Haikun Liu's lab) at 1/500, rabbit antiPTEN ( ab-31392, Abcam) at 1/1000, mouse anti-p21 (CS2948囚 CST) at 1/1000, mouse anti-ACTIN (sc47778, Santa Cruz) at 1/1000, mouse anti-GAPDH (sc-137179, Santa Cruz) at 1/1000. Suitable secondary antibodies, such as anti-rabbit or anti-mouse antibodies conjugated with HRP, were used for ECL detection (Amershan).

\section{Immunofluorescence staining}

The cells were fixed were $4 \%$ paraformaldehyde (PFA) for 30 minutes at 4 degree, and then washed in cold PBS for 5 minutes 3 times. The nuclei were subsequently permeabilized with PBS with $0.5 \%$ Triton X100 for 30 minutes. Next the cells were blocked with 1\% BSA in PBS for 1 hour. The cells were incubated with primary antibody overnight at $4^{\circ} \mathrm{C}$. Primary antibodies used were rabbit anti-cleaved caspase 3 antibody (9664S, CST) and rabbit anti-Ki67 (ab15580, Abcam). After wash, the cells were incubated with anti-rabbit secondary antibody conjugated with proper Alexa Fluor label for 1 hour at room temperature in darkness. The nuclei were counterstained with DAPI. The cells were imaged with Olympus IX-73 immunofluorescence microscope.

\section{Co-immunoprecipitation}

Immunoprecipitation assays were performed with whole-cell lysates from BTICs or targeted cells transfected with overexpression plasmids. Anti-NR2E1 (ab30942), anti-LSD1(ab17721), anti-FLAG (SIGMA \#F 1804) and anti-HA (ab18181) antibodies were used to pull down protein complexes. 
Immunoprecipitated complexes, bound to the corresponding antibody, were washed extensively with $0.1 \%$ Triton X-100 buffer (50 mM Tris-HCl at pH 8, $150 \mathrm{mM} \mathrm{NaCl}, 1 \mathrm{mM}$ EDTA, 0.1\% Triton X-100, 10\% glycerol plus Roche protease inhibitor cocktail). The interacting protein bands were resolved with 10\% SDS-PAGE gel and transferred to the PVDF membrane, followed by detection with an appropriate primary antibody, an HRP-conjugated second antibody, and an ECL detection reagent.

\section{Animals}

The animal experiments were performed under the approval from Animal Experimentation Ethics Committee (AEEC) in the Sixth Affiliated Hospital of Sun Yat-sen University. 1X105 GFP or LSD1-197-211GFP transduced human BTICs were intracranially transplanted into the frontal lobe of 6 to 8-week-old female nude mice. The cells were suspended in $5 \mathrm{ul} \mathrm{PBS}$ and injected into the right frontal lobe at $2 \mathrm{~mm}$ lateral and $1 \mathrm{~mm}$ anterior to bregma with $2.5 \mathrm{~mm}$ depth from the skull base. The mice were fed with water containing fresh $2 \mathrm{mg} / \mathrm{ml}$ Doxycycline (DOX) daily on day after transplantation. The brain tumor growth was monitored by IVIS Spectrum imaging (PerkinElmer) after transplantation. The mouse brains were harvested for hematoxylin-eosin staining.

\section{Amide Hydrogen/Deuterium Exchange and Mass Spectrometry (HDX-MS)}

Human NR2E1 (GenScript) was cloned into pET22b to express protein with C-terminal His tag. The protein was purified with Ni-NTA beads and followed by gel filtration. Human LSD1 (ATCC) was cloned into pGEX6 to express protein with N-terminal GST tag. The protein was purified with glutathione agarose and the GST tag was removed by precission protease digestion at 4 degree overnight. The eluted protein was further purified by gel filtration. To study the peptide of Lsd1 involved in the interaction with NR2E1, $50 \mu \mathrm{M}$ of full length human LSD1 protein was incubated with $75 \mu \mathrm{M}$ of human NR2E1 (residues 183-354) in buffer $\mathrm{A}(25 \mathrm{mM}$ potassium phosphate and $5 \%$ glycerol at $\mathrm{pH} 7.5)$ for $12 \mathrm{hrs}$ prior to the HDX experiments. $2 \mu \mathrm{L}$ of LSD1 alone or in complex with NR2E1 was mixed with $18 \mu \mathrm{L}$ of deuterated buffer $\mathrm{A}$ resulting in a final concentration of $90 \% \mathrm{D}_{2} \mathrm{O}$. Exchange reactions were carried out at $20^{\circ} \mathrm{C}$ for five different time points ( 0.5 to $10 \mathrm{~min}$ ) and quenched by adding $40 \mu \mathrm{L}$ of ice cold $0.1 \%$ trifluoro acetic acid to get a final pH of 2.5. $50 \mu \mathrm{L}$ aliquot of the quenched samples containing $0.83 \mu \mathrm{M}$ of Lsd1 was injected onto a chilled nano-Ultra Performance Liquid Chromatography sample manager (test version, Waters), specially designed for HDX experiments. Online digestion was carried out using an immobilized pepsin column (Porozyme, $\mathrm{ABI}$ ) in water containing $0.05 \% \mathrm{FA}$ at a flow rate of $100 \mu \mathrm{L} / \mathrm{min}$. The digested sample was desalted in a $2.1 \times 5 \mathrm{~mm}$ C18 peptide microtrap (ACQUITY BEH C18 VanGuard Pre-column, $1.7 \mu \mathrm{m}$, Waters) and eluted using a linear gradient of acetonitrile (8-40\%) in $0.1 \% \mathrm{FA}$, onto a reverse phase analytical column (ACQUITY UPLC BEH C18 Column, $1.0 \times 100 \mathrm{~mm}, 1.7 \mu \mathrm{m}$, Waters) at $40 \mu \mathrm{L} / \mathrm{min}$. Mass spectra were acquired over the $\mathrm{m} / \mathrm{z}$ range $200-1700$ and continuous instrument calibration was carried out using Glu-Fibrinogen peptide (GFP) at $100 \mathrm{fmol} / \mu \mathrm{l}$. Peptides were identified from MSE data of an undeuterated Lsd1 sample using ProteinLynx Global Server (PLGS 2.4) (test version, Waters) and mapped on to subsequent deuteration experiments using prototype custom software (DynamX, Waters). The average number of deuterons exchanged for each of the pepsin digest fragments was obtained as 
described before(Mandell et al., 1998). Exchange values were not corrected for the deuterium backexchange, that occurs during sample analysis and so all the results reported in this study are only from the relative deuterium level. The difference in deuterium uptake (subtracting the absolute deuterium level in LSD1 from the NR2E1:LSD1 samples) for 56 pepsin digest fragments at all the time points was plotted using Origin software (Origin Pro v.8.6, OriginLab). The percent difference in deuterium uptake for all of the pepsin digest fragments between LSD1 and NR2E1:LSD1 following 1 min HDX were shown below (Eqn. 1) and mapped onto the crystal structure of LSD1 (PDB ID: 2Z3Y) using PyMOL (PyMOL Molecular Graphics System, Version 1.3, Schrodinger, LLC).

\section{Percent difference \\ $=\frac{\text { Deuterium uptake by Lsd } 1-\text { Deuterium uptake by Lsd } 1: \text { Tlx }}{\text { Total number of exchangeable amides }} \times 100$}

\section{Crystal structure determination}

The SWIRM domain of human LSD1 residue 172 to 280 , was subcloned into the pET15b vector between the EcoRI and $\mathrm{BamH} 1$ restriction sites, and the resulting plasmid was transformed into E. coli strain BL21. Cells were grown at $37{ }^{\circ} \mathrm{C}$ in LB medium to an optical density of 0.6 at $600 \mathrm{~nm}$ and induced with $0.5 \mathrm{mM}$ IPTG. The collected cells were lysed by French press in a buffer containing $50 \mathrm{mM}$ Tris, pH 7.0, $150 \mathrm{mM}$ $\mathrm{NaCl}, 1 \mathrm{mM}$ EDTA and $1 \mathrm{mM}$ dithiothreitol (DTT). After removing cell debris by centrifugation at 10,000xg for 30 minutes, the supernatant was mixed with Ni-NTA resin, and then poured into a column. After extensive wash, the SWIRM domain protein was released from the resin by $300 \mathrm{mM}$ imidazole. Selenomethionine (SeMet) protein was expressed following the standard method (Yuan et al., 2003). Native crystals were grown at $20^{\circ} \mathrm{C}$ by the hanging drop vapor diffusion method: $2 \mu$ of protein at a concentration of $10 \mathrm{mg} \mathrm{ml}-1$ was mixed with equal amount of reservoir buffer consisting of $0.1 \mathrm{M}$ $\mathrm{MgCl}_{2}, 0.1 \mathrm{M}$ Tris pH 8.5, 25\% (w/v) PEG 3350. SeMet derivative crystals were grown in reservoir buffer containing $0.8 \mathrm{M} \mathrm{K} / \mathrm{Na}$ tartrate, $0.1 \mathrm{M}$ MES pH $6,2.5 \%$ (v/v) glycerol. Native SWIRM protein crystallizes in the space groups $\mathrm{P} 2{ }_{1} 2{ }_{1} 2_{1}$ and $\mathrm{P} 2{ }_{1} 2{ }_{1} 2$ whereas SeMet SWIRM protein crystallized in space group I222. Diffraction data of native crystals and SeMet crystals were collected at beamline A1 and beamline F2 respectively in Cornell High Energy Synchrotron Source. All data were processed with DENZO/SCALEPACK. The structure was determined by multiwavelength anomalous dispersion (MAD) methods using SHELX and SHARP and refined using CNS (Brunger et al., 1998). Native crystal structures were solved by molecular replacement with PHASER using the MAD structure as an initial model. There are two molecules in asymmetric unit. Pymol was used to calculate the cavity and binding pocket and draw the structure. The human LSD1 SWIRM domain coordinates have been deposited in the Protein Data Bank (Accession code 5IT3).

\section{Prediction of the interaction between Lsd1 and NR2E1 LBD}

ZDOCK V3.0.2 (http://zdock.umassmed.edu/) was used to predict the interaction between LSD1 (PDB code: 3ZMU) and NR2E1 LBD (PDB code: 4XAl) by rigid body docking (Chen et al., 2003). ZDOCK 
explicitly searches rotational space and uses a Fast Fourier Transformation (FFT) algorithm to speed up searching in translational spaces. Residues 256-333, 410-435, 575-626, 717-742 and 792-819 of LSD1, assumed to be not participating in the interaction between LSD1 and NR2E1 LBD were set as block residues. ZDOCK generated docking complexes were then clustered by the MMTSB clustering method (http://www.mmtsb.org) at $8 \AA$ RMSD cutoff (Feig et al., 2004). LSD1 peptide accessible areas on NR2E1 were calculated as buried solvent-accessible area with a $1.4 \AA$ probe radius using the Naccess software (Hubbard and Thornton, 1993).

\section{Declarations}

\section{Supplementary material}

Supplementary information of two tables and six figures is included as part of this submission.

\section{Ethics approval}

The animal experiments were performed under the approval from Animal Experimentation Ethics Committee (AEEC) in the Sixth Affiliated Hospital of Sun Yat-sen University.

\section{Conflict of interest statement}

The authors declare that no conflicts of interest exist.

\section{Funding}

This work was supported by funds from National Natural Science Foundation of China (NSFC) (Grant No. 81773156 to PY and Grant No. 8187232 to YM) and the support of the Ministry of Education of Singapore Academic Research Fund to the Singapore researchers to KS.

\section{Author contributions}

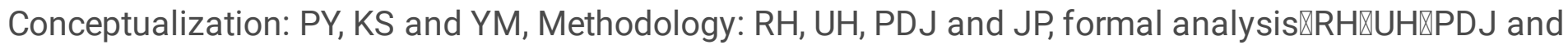
FC, investigation:RH, UH, XS, BSM, WZ, LZ, XM and PKG, resources: YM, writing of the original draft: PY,

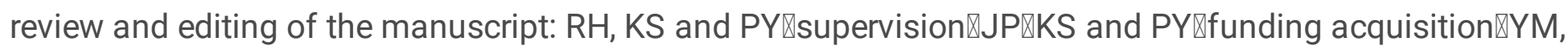
KS and PY.

\section{Acknowledgements}

We thank Haikun Liu from German Cancer Research Center for providing the brain tumor initiating cells and anti-Tlx antibody and Yanhong Shi from City of Hope for scientific discussion. We thank Yigong Shi from Xihu University for his support on SWIRM structure determination.

\section{Data availability statement}


The raw data that supports the findings of this study are available from the corresponding author upon reasonable request.

\section{References}

1. Baron, R., and Vellore, N.A. (2012). LSD1/CoREST is an allosteric nanoscale clamp regulated by H3histone-tail molecular recognition. Proc Natl Acad Sci U S A 109, 12509-12514.

2. Brunger, A.T., Adams, P.D., Clore, G.M., DeLano, W.L., Gros, P., Grosse-Kunstleve, R.W., Jiang, J.S., Kuszewski, J., Nilges, M., Pannu, N.S., et al. (1998). Crystallography \& NMR system: A new software suite for macromolecular structure determination. Acta Crystallogr D Biol Crystallogr 54, 905-921.

3. Chen, J., Li, Y., Yu, T.S., McKay, R.M., Burns, D.K., Kernie, S.G., and Parada, L.F. (2012). A restricted cell population propagates glioblastoma growth after chemotherapy. Nature 488, 522-526.

4. Chen, R., Li, L., and Weng, Z. (2003). ZDOCK: an initial-stage protein-docking algorithm. Proteins 52, 80-87.

5. Chen, Y., Yang, Y., Wang, F., Wan, K., Yamane, K., Zhang, Y., and Lei, M. (2006). Crystal structure of human histone lysine-specific demethylase 1 (LSD1). Proc Natl Acad Sci U S A 103, 13956-13961.

6. Da, G., Lenkart, J., Zhao, K., Shiekhattar, R., Cairns, B.R., and Marmorstein, R. (2006). Structure and function of the SWIRM domain, a conserved protein module found in chromatin regulatory complexes. Proc Natl Acad Sci U S A 103, 2057-2062.

7. Feig, M., Karanicolas, J., and Brooks, C.L., 3rd (2004). MMTSB Tool Set: enhanced sampling and multiscale modeling methods for applications in structural biology. J Mol Graph Model 22, 377-395.

8. Houshyari, M., Hajalikhani, F., Rakhsha, A., and Hajian, P. (2015). A Comparative Study of Survival Rate in High Grade Glioma Tumors Being Treated by Radiotherapy Alone Versus Chemoradiation With Nitrosourea. Glob J Health Sci 7, 43702.

9. Hubbard, S.J., and Thornton, J.M. (1993). NACCESS, Computer Program. . Department of Biochemistry Molecular Biology, University College London.

10. Kashyap, V., Ahmad, S., Nilsson, E.M., Helczynski, L., Kenna, S., Persson, J.L., Gudas, L.J., and Mongan, N.P. (2013). The lysine specific demethylase-1 (LSD1/KDM1A) regulates VEGF-A expression in prostate cancer. Mol Oncol 7, 555-566.

11. Lee, M.G., Wynder, C., Schmidt, D.M., McCafferty, D.G., and Shiekhattar, R. (2006). Histone H3 lysine 4 demethylation is a target of nonselective antidepressive medications. Chem Biol 13, 563-567.

12. Li, P., Chen, Y., Mak, K.K., Wong, C.K., Wang, C.C., and Yuan, P. (2013a). Functional role of mst1/mst2 in embryonic stem cell differentiation. PLoS One 8, e79867.

13. Li, P., Chen, Y., Meng, X., Kwok, K.Y., Huang, X., Choy, K.W., Wang, C.C., Lan, H., and Yuan, P. (2013b). Suppression of malignancy by Smad3 in mouse embryonic stem cell formed teratoma. Stem Cell Rev 9, 709-720.

14. Li, P., Ma, X., Adams, I.R., and Yuan, P. (2015). A tight control of Rif1 by Oct4 and Smad3 is critical for mouse embryonic stem cell stability. Cell Death Dis 6, e1588. 
15. Liang, Y., Quenelle, D., Vogel, J.L., Mascaro, C., Ortega, A., and Kristie, T.M. (2013). A novel selective LSD1/KDM1A inhibitor epigenetically blocks herpes simplex virus lytic replication and reactivation from latency. MBio 4, e00558-00512.

16. Lin, S., Shen, H., Li, J.L., Tang, S., Gu, Y., Chen, Z., Hu, C., Rice, J.C., Lu, J., and Wu, L. (2013).

Proteomic and functional analyses reveal the role of chromatin reader SFMBT1 in regulating epigenetic silencing and the myogenic gene program. The Journal of biological chemistry $288,6238-$ 6247.

17. Lynch, J.T., Harris, W.J., and Somervaille, T.C. (2012). LSD1 inhibition: a therapeutic strategy in cancer? Expert Opin Ther Targets 16, 1239-1249.

18. Mandell, J.G., Falick, A.M., and Komives, E.A. (1998). Measurement of amide hydrogen exchange by MALDI-TOF mass spectrometry. Anal Chem 70, 3987-3995.

19. Metzger, E., Wissmann, M., Yin, N., Muller, J.M., Schneider, R., Peters, A.H., Gunther, T., Buettner, R., and Schule, R. (2005). LSD1 demethylates repressive histone marks to promote androgen-receptordependent transcription. Nature 437, 436-439.

20. Mrugala, M.M. (2013). Advances and challenges in the treatment of glioblastoma: a clinician's perspective. Discov Med 15, 221-230.

21. Schenk, T., Chen, W.C., Gollner, S., Howell, L., Jin, L., Hebestreit, K., Klein, H.U., Popescu, A.C., Burnett, A., Mills, K., et al. (2012). Inhibition of the LSD1 (KDM1A) demethylase reactivates the all-transretinoic acid differentiation pathway in acute myeloid leukemia. Nat Med 18, 605-611.

22. Schmidt, D.M., and McCafferty, D.G. (2007). trans-2-Phenylcyclopropylamine is a mechanism-based inactivator of the histone demethylase LSD1. Biochemistry 46, 4408-4416.

23. Shi, Y., Chichung Lie, D., Taupin, P., Nakashima, K., Ray, J., Yu, R.T., Gage, F.H., and Evans, R.M. (2004a). Expression and function of orphan nuclear receptor TLX in adult neural stem cells. Nature 427, 78-83.

24. Shi, Y., Lan, F., Matson, C., Mulligan, P., Whetstine, J.R., Cole, P.A., and Casero, R.A. (2004b). Histone demethylation mediated by the nuclear amine oxidase homolog LSD1. Cell 119, 941-953.

25. Shi, Y.J., Matson, C., Lan, F., Iwase, S., Baba, T., and Shi, Y. (2005). Regulation of LSD1 histone demethylase activity by its associated factors. Mol Cell 19, 857-864.

26. Singh, M.M., Manton, C.A., Bhat, K.P., Tsai, W.W., Aldape, K., Barton, M.C., and Chandra, J. (2011). Inhibition of LSD1 sensitizes glioblastoma cells to histone deacetylase inhibitors. Neuro Oncol 13, 894-903.

27. Singh, S.K., Clarke, I.D., Terasaki, M., Bonn, V.E., Hawkins, C., Squire, J., and Dirks, P.B. (2003). Identification of a cancer stem cell in human brain tumors. Cancer Res 63, 5821-5828.

28. Singh, S.K., Hawkins, C., Clarke, I.D., Squire, J.A., Bayani, J., Hide, T., Henkelman, R.M., Cusimano, M.D., and Dirks, P.B. (2004). Identification of human brain tumour initiating cells. Nature 432, 396401.

29. Sun, G., Alzayady, K., Stewart, R., Ye, P., Yang, S., Li, W., and Shi, Y. (2010). Histone demethylase LSD1 regulates neural stem cell proliferation. Mol Cell Biol 30, 1997-2005. 
30. Thotala, D., Karvas, R.M., Engelbach, J.A., Garbow, J.R., Hallahan, A.N., DeWees, T.A., Laszlo, A., and Hallahan, D.E. (2015). Valproic acid enhances the efficacy of radiation therapy by protecting normal hippocampal neurons and sensitizing malignant glioblastoma cells. Oncotarget.

31. Tortorici, M., Borrello, M.T., Tardugno, M., Chiarelli, L.R., Pilotto, S., Ciossani, G., Vellore, N.A., Bailey, S.G., Cowan, J., O'Connell, M., et al. (2013). Protein recognition by short peptide reversible inhibitors of the chromatin-modifying LSD1/CoREST lysine demethylase. ACS Chem Biol 8, 1677-1682.

32. Weller, M., Gorlia, T., Cairncross, J.G., van den Bent, M.J., Mason, W., Belanger, K., Brandes, A.A., Bogdahn, U., Macdonald, D.R., Forsyth, P., et al. (2011). Prolonged survival with valproic acid use in the EORTC/NCIC temozolomide trial for glioblastoma. Neurology 77, 1156-1164.

33. Willmann, D., Lim, S., Wetzel, S., Metzger, E., Jandausch, A., Wilk, W., Jung, M., Forne, I., Imhof, A., Janzer, A., et al. (2012). Impairment of prostate cancer cell growth by a selective and reversible lysine-specific demethylase 1 inhibitor. Int J Cancer 131, 2704-2709.

34. Yokoyama, A., Takezawa, S., Schule, R., Kitagawa, H., and Kato, S. (2008). Transrepressive function of TLX requires the histone demethylase LSD1. Mol Cell Biol 28, 3995-4003.

35. Yuan, P., Jedd, G., Kumaran, D., Swaminathan, S., Shio, H., Hewitt, D., Chua, N.H., and Swaminathan, K. (2003). A HEX-1 crystal lattice required for Woronin body function in Neurospora crassa. Nat Struct Biol 10, 264-270.

36. Zhi, X., Zhou, X.E., He, Y., Searose-Xu, K., Zhang, C.L., Tsai, C.C., Melcher, K., and Xu, H.E. (2015). Structural basis for corepressor assembly by the orphan nuclear receptor TLX. Genes Dev 29, 440450.

37. Zhu, Z., Khan, M.A., Weiler, M., Blaes, J., Jestaedt, L., Geibert, M., Zou, P., Gronych, J., Bernhardt, O., Korshunov, A., et al. (2014). Targeting self-renewal in high-grade brain tumors leads to loss of brain tumor stem cells and prolonged survival. Cell Stem Cell 15, 185-198.

\section{Figures}


Fig. 1

a

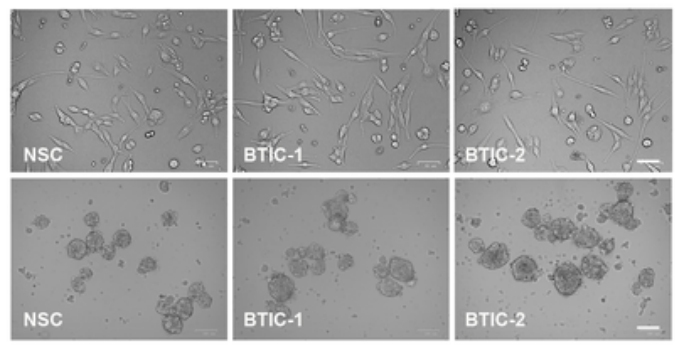

C

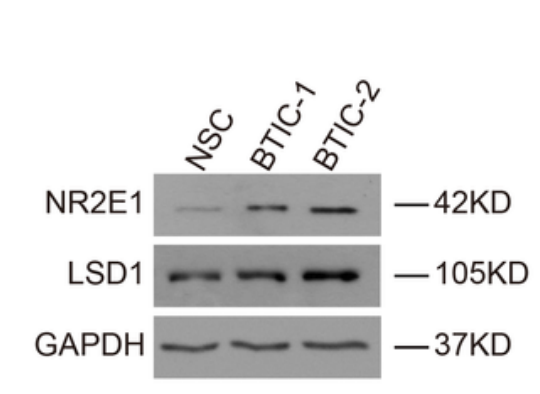

e
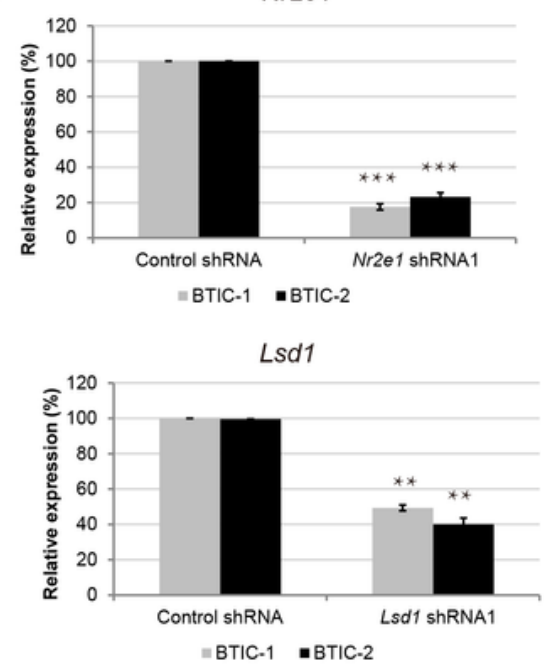

d

f b
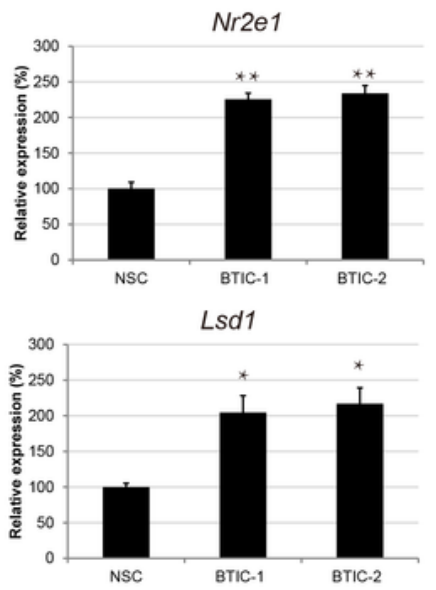
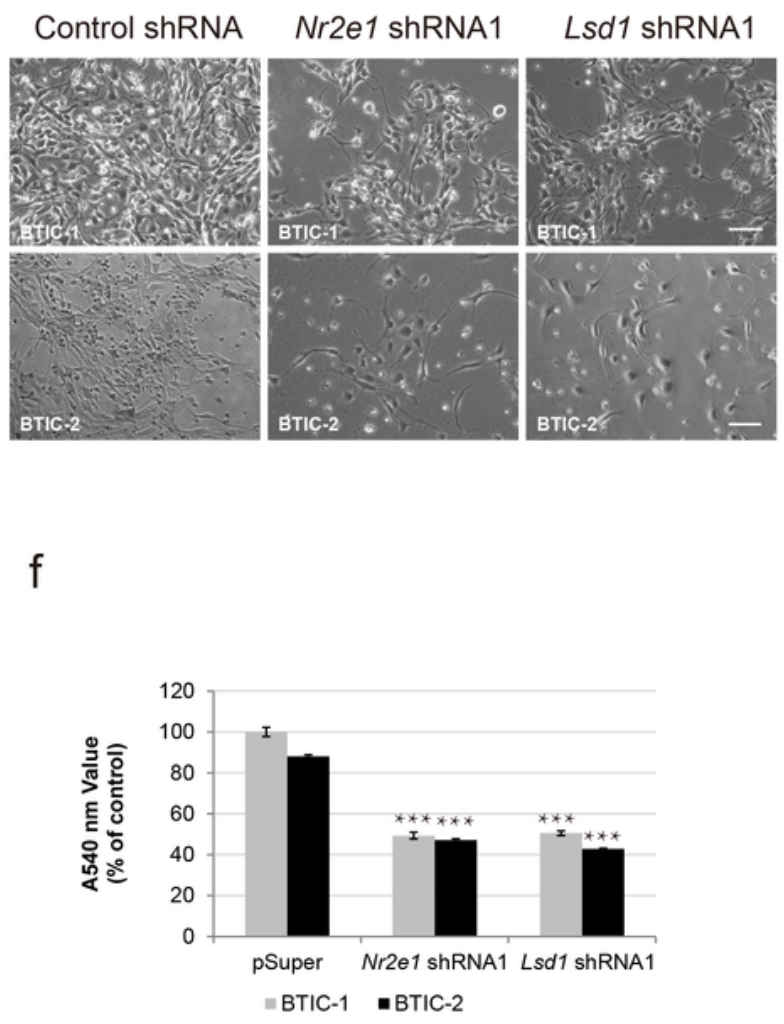

\section{Figure 1}

NR2E1 and LSD1 are required for the proliferation of BTICs. a. Morphology of neural stem cells (NSCs) and two lines of brain tumor initiating cells (BTIC-1 and BTIC-2) cultured in monolayer (top panel) and suspension (bottom panel). The scale bar is $50 \mu \mathrm{m}$. b. Real-time PCR analysis of mRNA level of Nr2e1 and Lsd1 in NSCs and BTICs. The expression data was normalized with actin. Data are represented as mean \pm S.D. $(n=3)$. Statistically significant differences, calculated through student's t-test, are indicated 
$\left({ }^{*} \mathrm{P}<0.05 ;{ }^{*} \mathrm{P}<0.005\right) . \mathrm{c}$. Western blot of the protein level of NR2E1 and LSD1 in NSCs and BTICs. GAPDH was used as an internal loading control. d. Cell morphology of BTICs after knockdown by control shRNA, Nr2e1 shRNA1 and Lsd1 shRNA1. The scale bar is $50 \mu \mathrm{m}$. e. Real-time PCR analysis of mRNA level of Nr2e1 and Lsd1 in BTICs after knockdown by control shRNA, Nr2e1 shRNA1 and Lsd1 shRNA1. Data are represented as mean \pm S.D. $(n=3)$. Statistically significant differences, calculated through

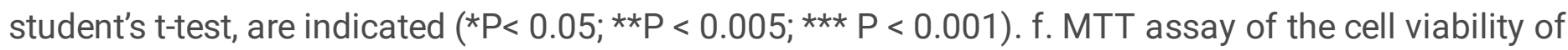
BTICs after knockdown with control shRNA, Nr2e1 shRNA1 or Lsd1 shRNA1. Data are represented as mean \pm S.D. $(n=3)$. Statistically significant differences, calculated through student's t-test, are indicated (*P< 0.05; **P $<0.005 ; * \star \star ~ P<0.001)$. 
Figure 2

a

IB: Light chain

Lysate: GAPDH

Lorem ipsum

C

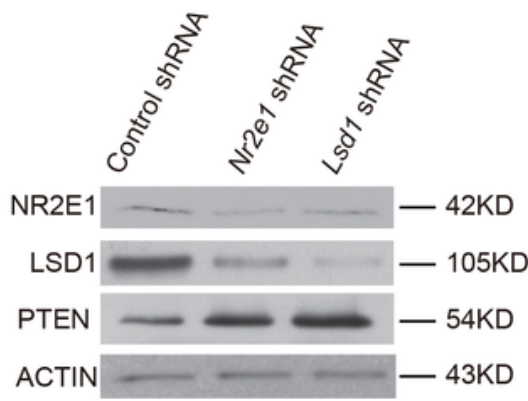

e
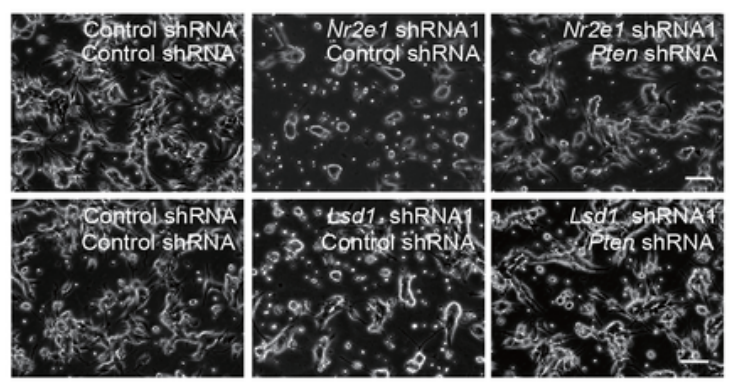

g
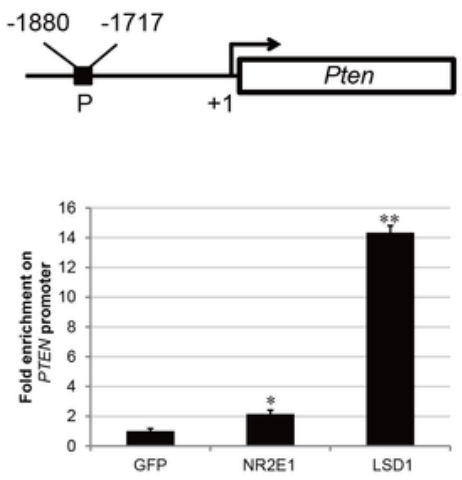

b

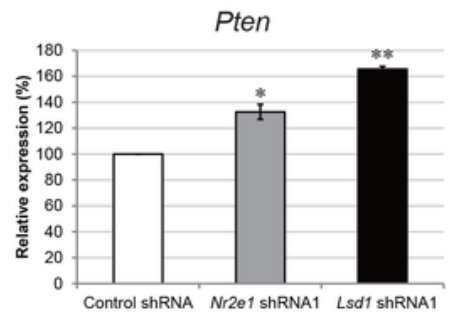

d

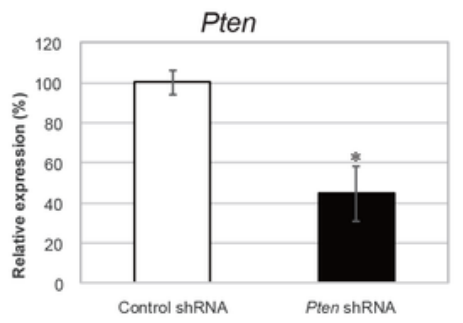

f

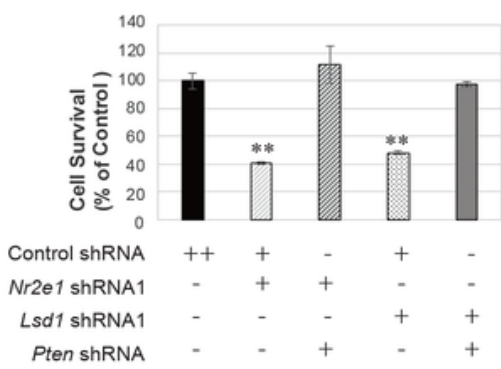

h
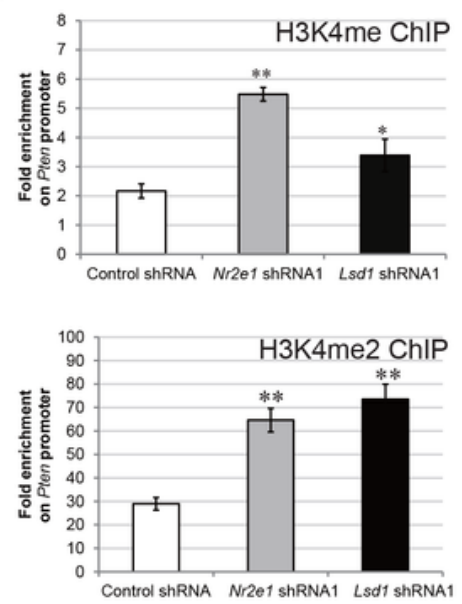

Figure 2

NR2E1 and LSD1 form a complex in BTICs and regulate PTEN expression. a. Co-immunoprecipitation (Co-IP) assay with antibodies against NR2E1 and LSD1 to test their interaction in BTICs. Equal amount of total cell lysate was immunoprecipitated with control IgG and an antibody against NR2E1. The pulled down proteins were then blotted with an anti-LSD1 antibody. GAPDH in the lysate was shown as a control for the input material for each Co-IP assay. IgG light chain was shown as a loading control of Co-IP pull- 
down material. b. Real-time PCR analysis of mRNA level of Pten in BTIC after control shRNA, Nr2e1 shRNA1 or Lsd1 shRNA1 knockdown. Data are represented as mean \pm S.D. $(n=3)$. Statistically significant differences, calculated through student's t-test, are indicated ( $\left.{ }^{\star} P<0.05 ; * \star P<0.005\right)$. c. Western blot analysis of the protein level of NR2E1, LSD1 and PTEN in BTICs after control shRNA, Nr2e1 shRNA or Lsd1 shRNA knockdown. Actin was used as an internal control. d. Real-time PCR analysis of mRNA level of Pten in BTICs after control shRNA or Pten shRNA knockdown. Data are represented as mean \pm S.D. $(n=$ 3). Statistically significant differences, calculated through student's t-test, are indicated $\left({ }^{\star} P<0.05\right)$. e. Cell morphology of BTICs after control shRNA, Nr2e1 shRNA1, Lsd1 shRNA1 and Pten shRNA knockdown as indicated combination. Scale bar represents $100 \mu \mathrm{m}$. f. Cell survival analysis of BTICs after control shRNA, Nr2e1 shRNA1, Lsd1 shRNA1 and Pten shRNA knockdown as indicated combination using cell counting kit-8. Data are represented as mean \pm S.D. $(n=3)$. Statistically significant differences, calculated through student's t-test, are indicated $\left({ }^{*} P<0.05 ; * * P<0.005\right)$. g. Scheme depicting the region where primers were designed for amplification of ChIP DNA and relative enrichment of control GFP, LSD1 and NR2E1 at Pten promoter revealed by Chromatin immunoprecipitation (ChIP) and real-time PCR assay. Data are represented as mean \pm S.D. $(n=3)$. Statistically significant differences, calculated through student's t-test, are indicated ( $\left.{ }^{*} P<0.05 ;{ }^{*} \mathrm{P}<0.005\right)$. h. ChIP and real-time PCR analysis of H3K4me1 and H3K4me2 enrichment at Pten promoter in BTICs after knockdown by control shRNA, Nr2e1 shRNA or Lsd1 shRNA. Data are represented as mean \pm S.D. $(n=3)$. Statistically significant differences, calculated through student's t-test, are indicated $\left({ }^{*} \mathrm{P}<0.05 ;{ }^{* * \mathrm{P}}<0.005\right)$. 
Figure 3

a

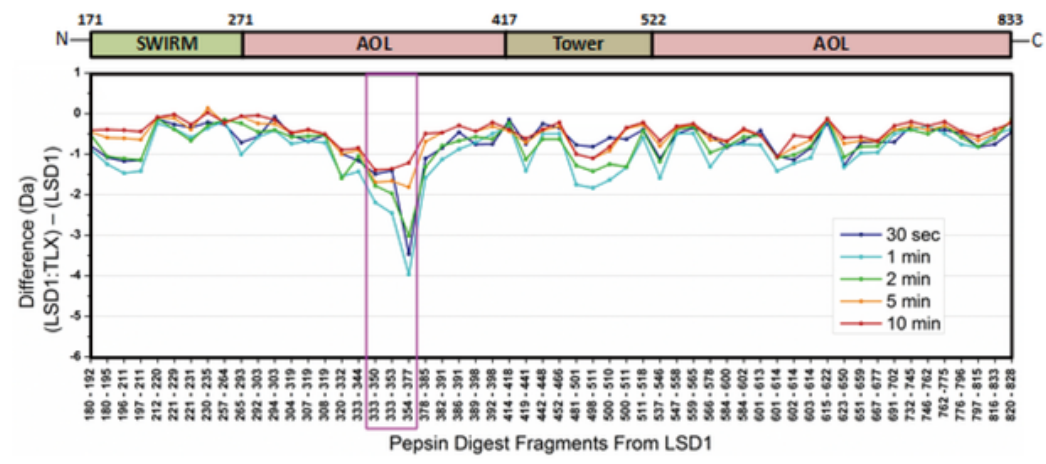

$\mathrm{b}$

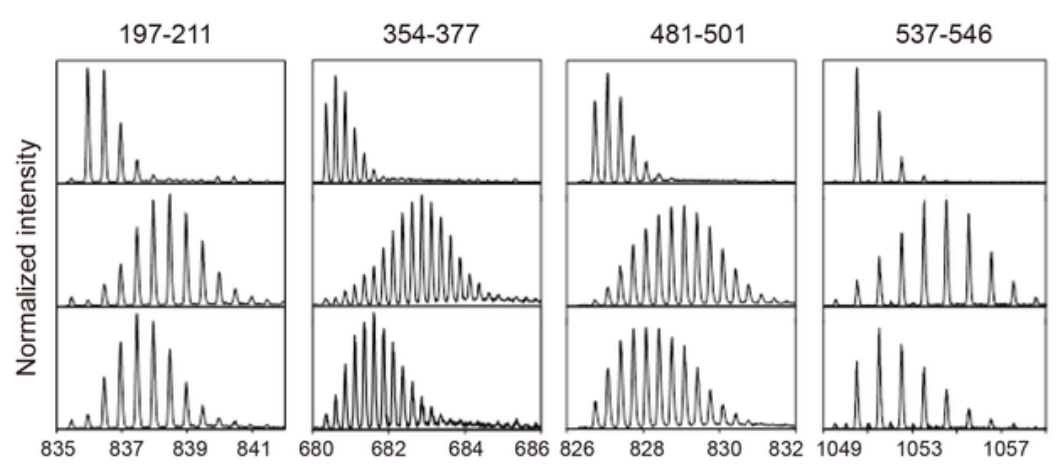

$\mathrm{c}$

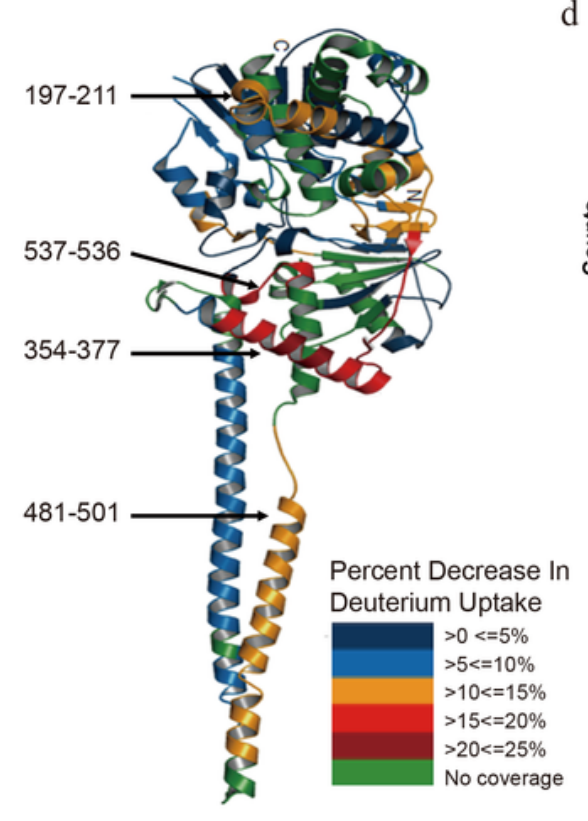

d

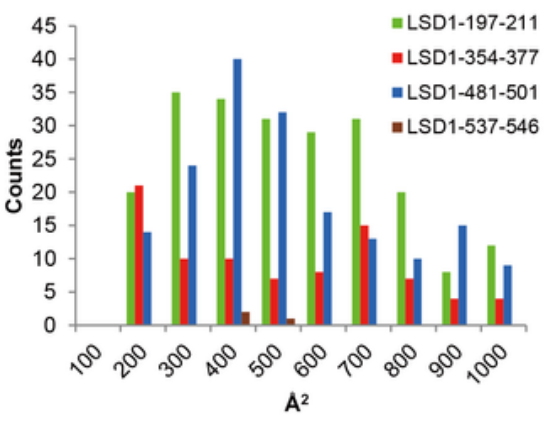

Figure 3

Effect of NR2E1 on LSD1 as shown by HDX-MS. a. The difference in absolute deuterium uptake between LSD1 and NR2E1:LSD1. b. Enhanced mass spectra for four pepsin digest fragments of LSD1, 197-211, 354-377, 481-501 and 537-546 which show significant differences in exchange upon NR2E1 binding: undeuterated peptide (top), isotopic envelope for the same peptide from LSD1 alone following 1 min deuteration (middle) and isotopic envelope for the same peptide from LSD1 in complex with NR2E1 
following 1 min deuteration (bottom). c. Heat map showing the percent decrease in deuterium uptake for NR2E1:LSD1 relative to LSD1 following 1 min of HDX, mapped on the crystal of LSD1 (PDB ID: 2Z3Y). d. The histogram plot of the distribution of the surface area of NR2E1 buried by LSD1 peptides 197-211, 354-377, 481-501 and 537-546. (Buried surface area less than $100 \AA 2$ is neglected).

Figure 4

a

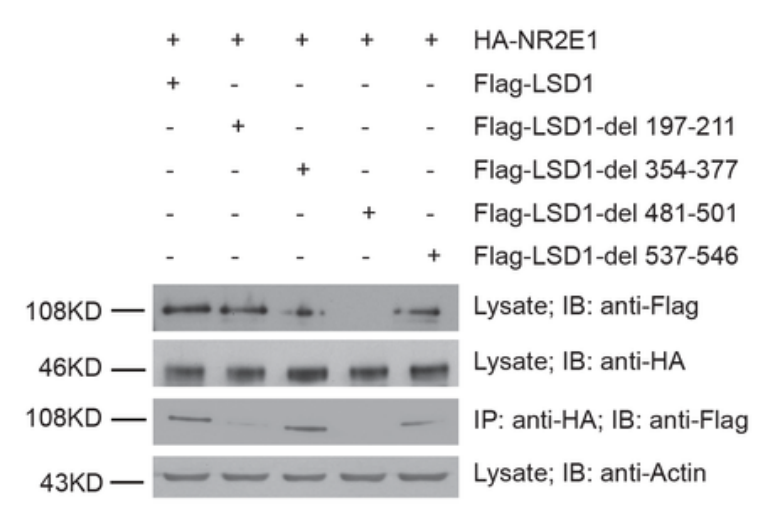

C
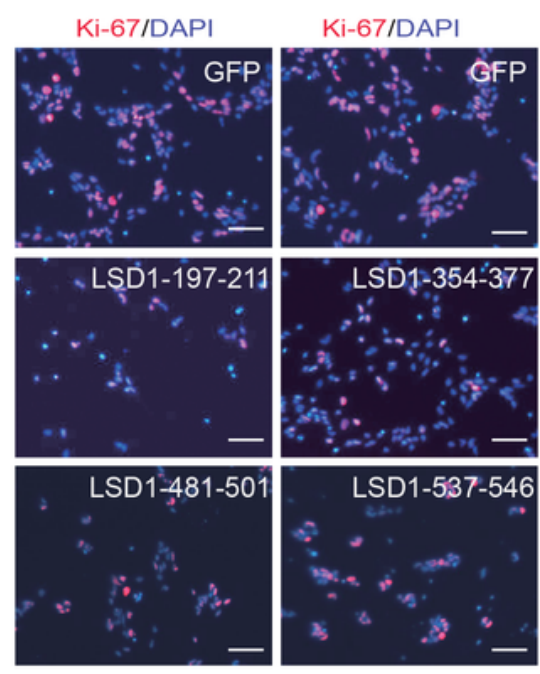

g

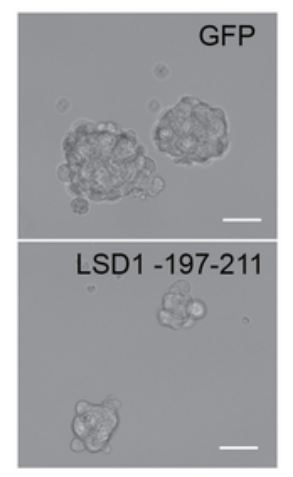

$\mathrm{h}$

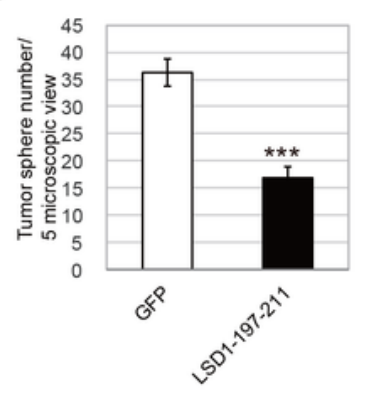

b

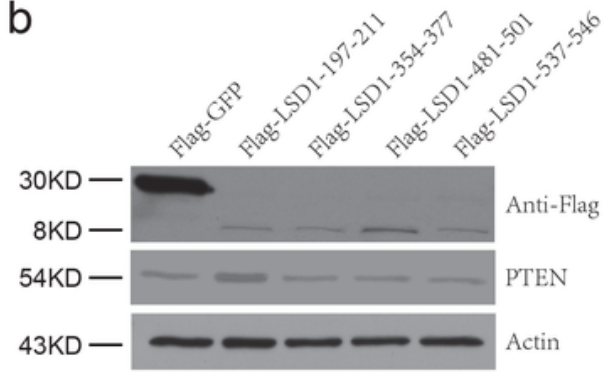

d
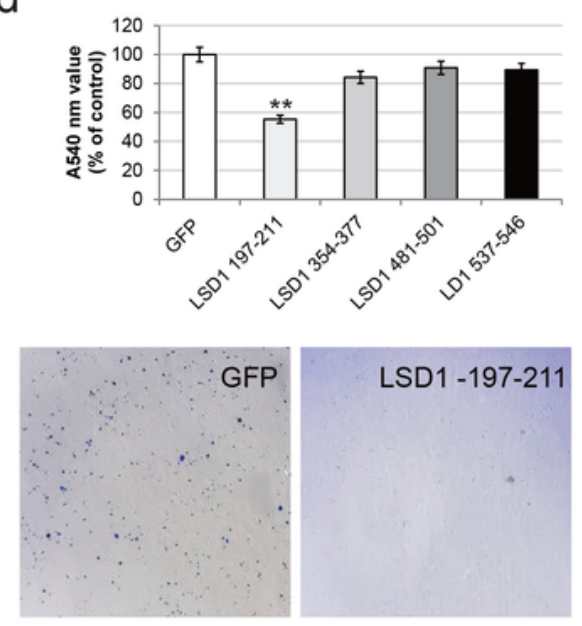

LSD1 -197-211

f
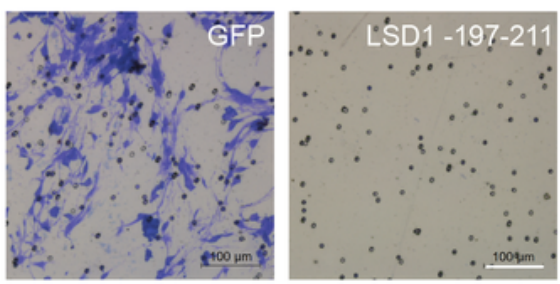

i

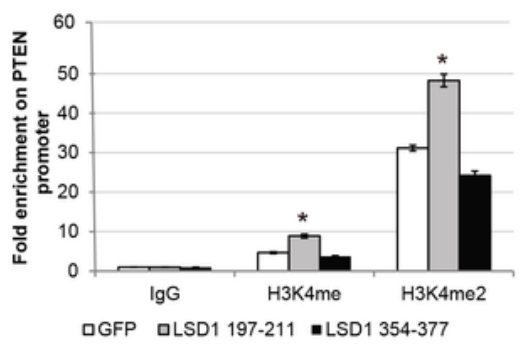

Figure 4 
The role of LSD1 peptides on NR2E1 and LSD1 interaction. a. The interaction between NR2E1 and Lsd1 was disrupted by the deletion of residues 197-211 in LSD1. Total protein lysate of HA-NR2E1 and FlagLSD1 or Flag-LSD1 mutant transfected 293T cells were immunoprecipitated with anti-HA antibody, followed by immunoblotting with anti-Flag antibody. Protein expression in the cell was shown by immunoblotting with anti-Flag or anti-HA antibody. b. Overexpression of Flag-LSD1 peptide 197-211 led to the upregulation of pTEN in BTICs. Lysates of Flag-GFP and Flag-LSD1 peptide overexpressed BTICs were immunoblot by anti-Flag and anti- PTEN antibodies. Actin was loaded as an internal loading control. c. Overexpression of LSD1 peptide 197-211 led to a reduction of Ki-67 positive cells. Anti-Ki-67 antibody staining was shown in red and DAPI staining was shown in blue. The scale bar is $25 \mu \mathrm{m}$. d. MTT assay of the viable BTICs after transient overexpression of control or LSD1 peptides. Data are represented as mean \pm S.D. $(n=4)$. Statistically significant differences, calculated through student's t-test, are indicated $\left(\star \star P<0.005 ;{ }^{* \star *} \mathrm{P}<0.001\right)$. e. Representative picture of soft agar assay of BTICs after overexpression of control peptide or LSD1-197-211 peptide. $f$. Representative picture of transwell assay to show the migration capacity of GFP overexpressed BTICs and LSD1-197-211 overexpressed BTICs. Scale bar represents $100 \mu \mathrm{m}$. g. Representative morphology of suspension culture of BTICs after overexpression of control peptide or LSD1-197-211 peptide. Scale bar represents $50 \mu \mathrm{m}$. h. Diagram of tumor sphere number of BTICs after overexpression of control peptide or LSD1-197-211 peptide in random 5 microscopic view. Data are represented as mean \pm S.D. $(n=3)$. Statistically significant differences, calculated through student's t-test, are indicated ( $\left.{ }^{\star *} \mathrm{P}<0.005 ; \star \star \star P<0.001\right)$. i. Relative enrichment of H3K4me1 and H3K4me2 on Pten promoter after overexpression of GFP or LSD1-197-211 peptide in BTICs. Data are represented as mean \pm S.D. $(n=3)$. Statistically significant differences, calculated through student's t-test, are indicated ( $\left.{ }^{\star} P<0.05 ;{ }^{* \star} P<0.005\right)$. 
Figure 5

a

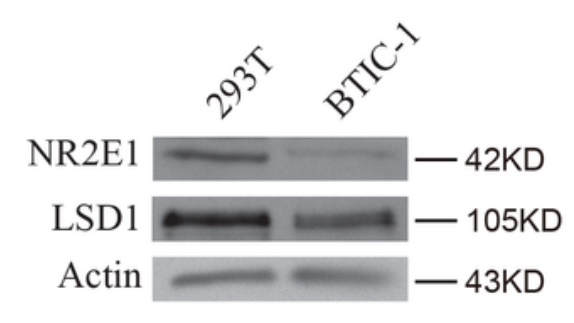

b

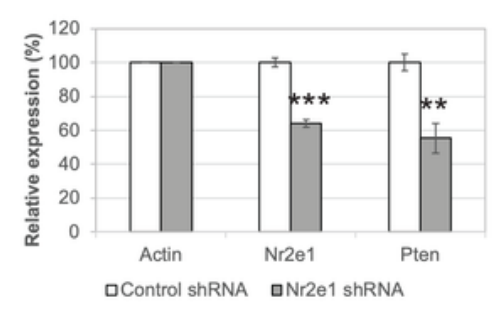

d

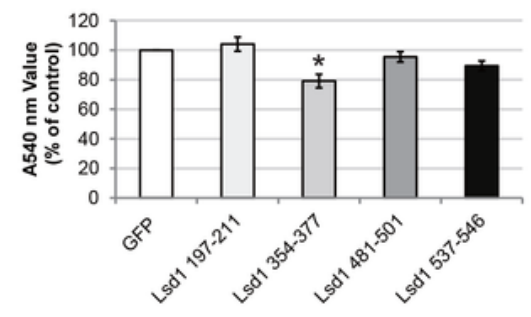

e

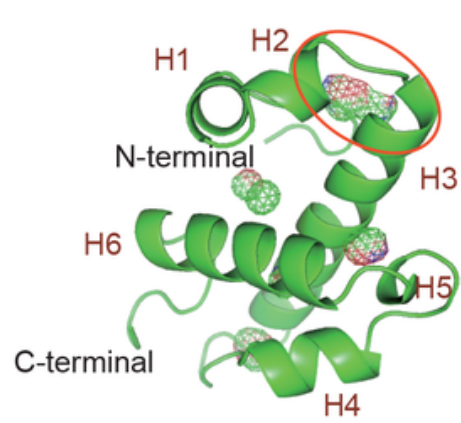

LSD1 Swirm

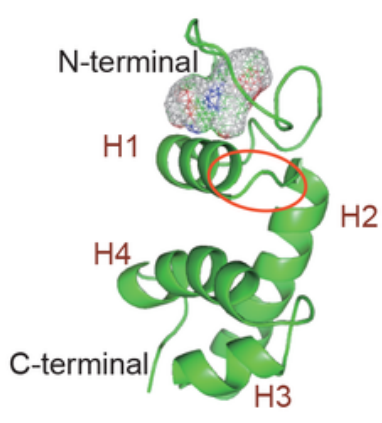

ADA2 Swirm

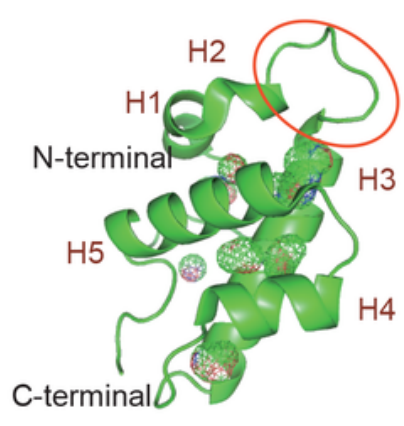

SWI3 Swirm

\section{Figure 5}

Specificity of LSD1-197-211. a. Western blot to check the protein level of NR2E1 and LSD1 in 293T and BTIC-1. Actin was loaded as an internal control. b. Real-time PCR quantification of the mRNA levels of Pten and Nr2e1 in control shRNA and Nr2e1 shRNA knockdown 293T cells. The data were normalized with Actin. Data are represented as mean \pm S.D. $(n=3)$. Statistically significant differences, calculated through student's t-test, are indicated $\left({ }^{*} P<0.05 ;{ }^{*} \mathrm{P}<0.005\right)$. c. Western blot to compare the expression 
level of PTEN in different LSD1 peptide overexpression BTICs and control GFP overexpression BTICs. GAPDH was loaded as an internal control. d. MTT assay of the cell viability of 293T cells after overexpression with control or LSD1 peptides. Data are represented as mean \pm S.D. $(n=3)$. Statistically significant differences, calculated through student's t-test, are indicated $\left({ }^{*} P<0.05\right)$. e. Overall structures of LSD1 SWIRM, ADA2 SWIRM (PDB Id: 2AQE) and SWI3 SWIRM (PDB ID: 2FQ3). All SWIRM domains contain a central long helix, flanked by small helices. LSD1-197-211 and the corresponding region in the ADA2 and SWI3 SWIRM domains are circled. Cavity/binding pocket is represented as mesh map. The Figures are drawn with Pymol. 
Figure 6

a

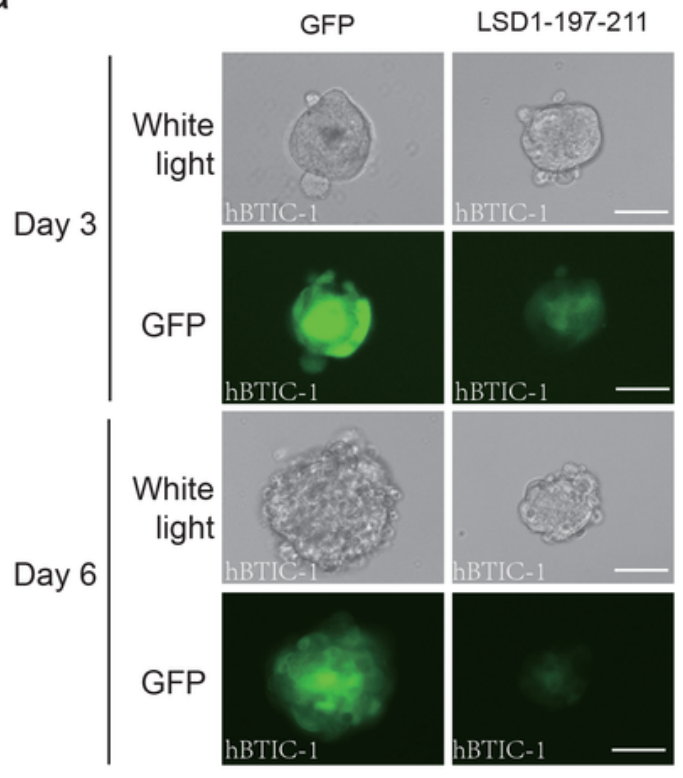

C

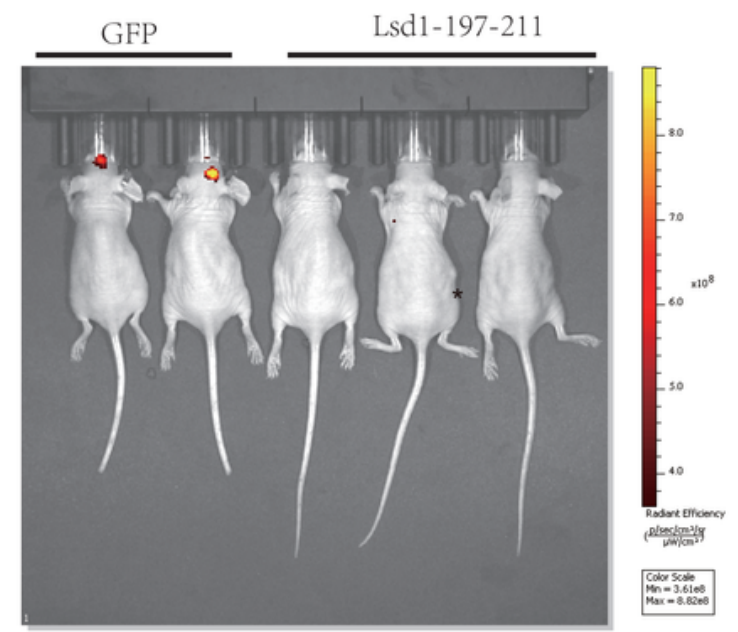

b
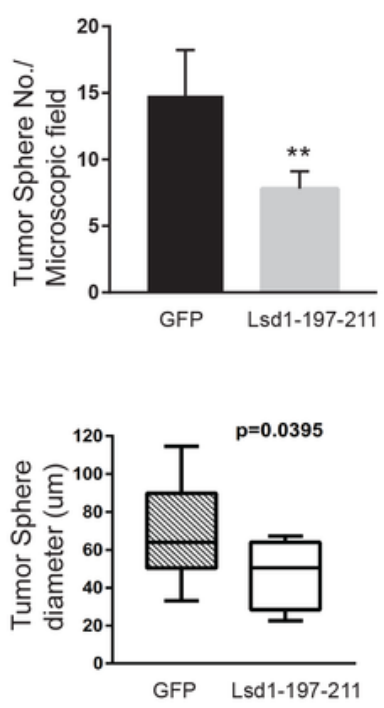

d

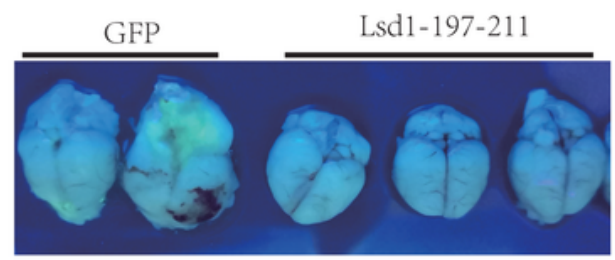

e GFP Lsd1-197-211
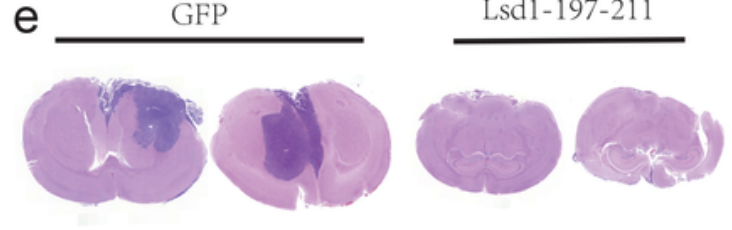

f
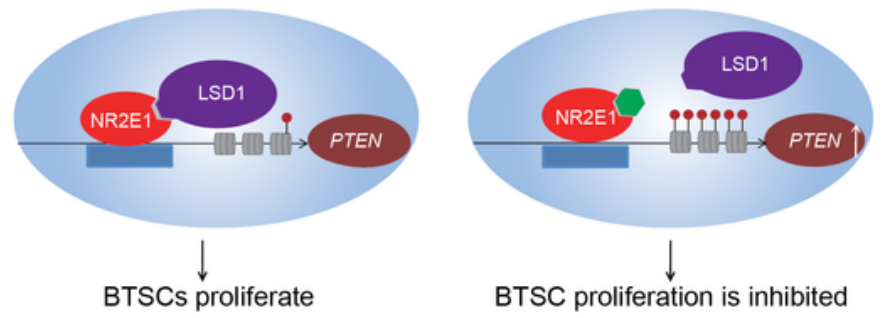

LSD1-197-211

i H3K4Me /H3K4me2

\section{Figure 6}

Stable expression of LSD1-197-211 inhibits the proliferation of human BTICs. a. Representative pictures of suspension culture of human BTIC line 1 (hBTIC-1) transduced by lentivirus that express GFP or LSD1197-211-GFP after induction by doxycycline for three days and six days. Light microscopy (Left), fluorescence microscopy to detect GFP (Right). Scale bar represents $50 \mu \mathrm{m}$. b. Diagram of tumor sphere number of hBTIC-1 transduced by lentivirus that express GFP or LSD1-197-211-GFP after induction by 
doxycycline for six days. Data are represented as mean \pm S.D. $(n=3)$. Statistically significant differences, calculated through student's t-test, are indicated $\left({ }^{*} P<0.05 ;{ }^{*} P<0.005\right)$. c. Representative IVIS images of brain tumors in nude mice transplanted with GFP or LSD1-197-211-EGFP lentivirus transduced human BTICs after five weeks of doxycycline induction d. Brains of nude mice transplanted with GFP or LSD1197-211-GFP lentivirus transduced human BTICs after seven weeks of doxycycline induction. e. Representative hematoxylin-eosin staining pictures of brains of nude mice transplanted with GFP or LSD1-197-211-GFP lentivirus transduced human BTICs after seven weeks of doxycycline induction. $\mathrm{f}$. A model to depict the functional mechanism of NR2E1, LSD1 and LSD1-197-211 in BTICs.

\section{Supplementary Files}

This is a list of supplementary files associated with this preprint. Click to download.

- Supplementarydocument.pdf 